\title{
An Entropic Perturbation Approach to TV-Minimization for Limited-Data Tomography
}

\author{
Andreea Deniţiu ${ }^{1,2}$, Stefania Petra ${ }^{1}$, Claudius Schnörr ${ }^{2}$, \\ and Christoph Schnörr ${ }^{1}$ \\ 1 Image and Pattern Analysis Group, University of Heidelberg, Germany \\ \{denitiu, petra, schnoerr\}@math. uni-heidelberg.de \\ 2 Hochschule München, Fakultät für Informatik und Mathematik, \\ München, Germany \\ denitiu@hm.edu, schnoerr@cs.hm.edu
}

\begin{abstract}
The reconstruction problem of discrete tomography is studied using novel techniques from compressive sensing. Recent theoretical results of the authors enable to predict the number of measurements required for the unique reconstruction of a class of cosparse dense $2 \mathrm{D}$ and $3 \mathrm{D}$ signals in severely undersampled scenarios by convex programming. These results extend established $\ell_{1}$-related theory based on sparsity of the signal itself to novel scenarios not covered so far, including tomographic projections of 3D solid bodies composed of few different materials. As a consequence, the large-scale optimization task based on total-variation minimization subject to tomographic projection constraints is considerably more complex than basic $\ell_{1}$-programming for sparse regularization. We propose an entropic perturbation of the objective that enables to apply efficient methodologies from unconstrained optimization to the perturbed dual program. Numerical results validate the theory for large-scale recovery problems of integer-valued functions that exceed the capacity of the commercial MOSEK software.
\end{abstract}

Keywords: discrete tomography, compressed sensing, underdetermined systems of linear equations, cosparsity, phase transitions, total variation, entropic perturbation, convex duality, convex programming.

\section{Introduction}

This paper addresses the problem of reconstructing compound solid bodies $u$ from few tomographic projections: $A u=b$. Theoretical guarantees of reconstruction performance relate to current research in the field of compressive sensing, concerned with real sensor matrices $A$ that do not satisfy commonly made assumptions like the restricted isometry property.

By adopting the cosparse signal model [1] that conforms to our tomographic scenario, the authors [2] recently established the existence of "phase transitions" that relate the required limited(!) number of measurements to the cosparsity level of $u$ in order to guarantee unique recovery. This result was extensively validated

E. Barcucci et al. (Eds.): DGCI 2014, LNCS 8668, pp. 262-274 2014.

(c) Springer International Publishing Switzerland 2014 
numerically using the commercial MOSEK software, to avoid that numerical optimization issues affect the validation.

For our 3D problems, a dedicated numerical optimization algorithm is necessary, however, because MOSEK cannot handle medium and large problem sizes. We present in this paper such an approach by utilizing the fact that adequate perturbations of the optimization problem may lead to a simpler dual problem [34]. We work out a corresponding approach to our specific reconstruction problem

$$
\min _{u} \operatorname{TV}(u) \text { subject to } A u=b \text {, }
$$

that minimizes the total variation $\mathrm{TV}(u)$ subject to the projection constraints.

Organization. Section 2 briefly reports the above-mentioned phase transitions, followed by presenting a reformulation in Section 3 together with uniqueness results. A suitable perturbation is worked out in Section 4 that favorably compares to MOSEK for relevant problem sizes (Section 5).

Basic Notation. For $n \in \mathbb{N}$, we denote $[n]=\{1,2, \ldots, n\}$. The complement of a subset $J \subset[n]$ is denoted by $J^{c}=[n] \backslash J$. For some matrix $A$ and a vector $z, A_{J}$ denotes the submatrix of rows indexed by $J$, and $z_{J}$ the corresponding subvector. $A_{i}$ will denote the $i$ th row of $A . \mathcal{N}(A)$ and $\mathcal{R}(A)$ denote the nullspace and the range of $A$, respectively. Vectors are column vectors and indexed by superscripts. Sometimes, we will write e.g. $(u, v)^{\top}$ instead of the more correct $\left(u^{\top}, v^{\top}\right)^{\top} \cdot A^{\top}$ denotes the transposed of $A . \mathbb{1}=(1,1, \ldots, 1)^{\top}$ denotes the onevector whose dimension will always be clear from the context. The dimension of a vector $z$ is $\operatorname{dim}(z)$. $\langle x, z\rangle$ denotes the standard scalar product in $\mathbb{R}^{n}$ and we write $x \perp z$ if $\langle x, z\rangle=0$. The number of nonzeros of a vector $x$ is denoted by $\|x\|_{0}$. The indicator function of a set $C$ is denoted by $\delta_{C}(x):=\left\{\begin{array}{ll}0, & \text { if } x \in C \\ +\infty, & \text { if } x \notin C\end{array}\right.$. $\sigma_{C}(x):=\sup _{y \in C}\langle y, x\rangle$ denotes the support function of a nonempty set $C . \partial f(x)$ is the subdifferential of $f$ at $x$ and int $C$ and $\operatorname{rint} C$ denote the interior and the relative interior of a set $C$. By $f^{*}$ we denote the conjugate function of $f$. We refer to [5] for related properties.

In what follows, we will work with an anisotropic discretization of the TV operator in (1) given by

$$
\mathrm{TV}(u):=\|B u\|_{1}, \quad B:=\left(\begin{array}{l}
\partial_{1} \otimes I \otimes I \\
I \otimes \partial_{2} \otimes I \\
I \otimes I \otimes \partial_{3}
\end{array}\right) \in \mathbb{R}^{p \times n},
$$

where $\otimes$ denotes the Kronecker product and $\partial_{i}, i=1,2,3$, are derivative matrices forming the forward first-order differences of $u$ wrt. the considered coordinates. Implicitly, it is understood that $u$ on the r.h.s. of (2) is a vector representing all voxel values in appropriate order.

\section{Weak Phase Transitions for TV-Based Reconstruction}

We summarize in this section an essential result from [2] concerning the unique recovery from tomographic projection by solving problem (1), depending on the 
cosparsity level $\ell$ of $u$ (Definition 1) and the number $m$ of measurements (projections) of $u$. This result motivates the mathematical programming approach discussed in Section 3 and the corresponding numerical optimization approach presented in Section 4 .

Definition 1 (cosparsity, cosupport). The cosparsity of $u \in \mathbb{R}^{n}$ with respect to $B \in \mathbb{R}^{p \times n}$ is

$$
\ell:=p-\|B u\|_{0}
$$

and the cosupport of $u$ with respect to $B$ is

$$
\Lambda:=\left\{r \in[p]:(B u)_{r}=0\right\}, \quad|\Lambda|=\ell .
$$

Thus, $B_{\Lambda} u=0$. In view of the specific operator $B$ given by (2), $\ell$ measures the homogeneity of the volume function $u$. A large value of $\ell$ can be expected for $u$ corresponding to solid bodies composed of few homogeneous components.

Proposition 1 ([2, Cor. 4.6]). For given $A \in \mathbb{R}^{m \times n}$ and $B \in \mathbb{R}^{p \times n}$, suppose the rows of $\left(\begin{array}{l}A \\ B\end{array}\right)$ are linearly independent. Then a $\ell$-cosparse solution $u$ to the measurement equations $A u=b$ will be unique if the number of measurements satisfies

$$
\begin{array}{ll}
m \geq 2 n-(\ell+\sqrt{2 \ell+1}-1) & \text { (in 2D) } \\
m \geq 2 n-\frac{2}{3}\left(\ell+\sqrt[3]{3 \ell^{2}}+2 \sqrt[3]{\frac{\ell}{3}}-2\right) & \text { (in 3D) } .
\end{array}
$$

The above lower bounds on the number of measurements $m$ required to recover a $\ell$-cosparse vector $u$, imply that recovery can be carried out by solving $\min _{u}\|B u\|_{0}$ subject to $A u=b$. Replacing this objective by the convex relaxation (2) yields an excellent agreement of empirical results with the prediction (5), as shown in [2], although the independency assumption made in Prop. (11) does not strictly hold for the sensor matrices $A$ and the operator $B$ (2) used in practice.

This motivates to focus on efficient and sparse numerical optimization techniques that scale up to large problem sizes.

\section{TV-Recovery by Linear Programming}

We consider the discretized TV-term (2), an additional nonnegative constraint on image $u$ and express $B u=z$. Thus, (1) becomes

$$
\min _{u, z}\|z\|_{1} \quad \text { s.t. } \quad B u=z, \quad A u=b, \quad u \geq 0 .
$$




\subsection{Primal Linear Program and Its Dual}

By splitting the variable $z$ in its positive $v^{1}:=\max \{0, z\}$ and negative part $v^{2}:=-\min \{0, z\}$ we convert problem (6) into a linear program in normal form. With

$$
M:=\left(\begin{array}{ccc}
B & -I & I \\
A & 0 & 0
\end{array}\right), \quad q:=\left(\begin{array}{l}
0 \\
b
\end{array}\right)
$$

and the polyhedral set

$$
\mathcal{P}:=\left\{x \in \mathbb{R}^{n+2 p}: M x=q, x \geq 0\right\}, \quad x:=\left(u, v^{1}, v^{2}\right)^{\top},
$$

problem (6) becomes the linear program $(P)$

$$
\text { (P) } \quad \min _{x \in \mathcal{P}}\langle c, x\rangle=\min _{\left(u, v^{1}, v^{2}\right) \in \mathcal{P}}\left\langle\mathbb{1}, v^{1}+v^{2}\right\rangle, \quad c=(0, \mathbb{1}, \mathbb{1})^{\top} .
$$

We further assume that $\mathcal{P} \neq \emptyset$, i.e. a feasible solution always exists. Due to $c \geq 0$, the linear objective in $(P)$ is bounded on $\mathcal{P}$. Thus $(P)$ always has a solution under the feasibility assumption. In view of basic linear programing theory, compare [5, 11.43], the dual program also has a solution. The dual program $(D)$ reads

$$
\text { (D) } \quad \min _{y}-\langle q, y\rangle, \quad M^{\top} y \leq c .
$$

With

$$
y=\left(\begin{array}{l}
y_{0} \\
y_{b}
\end{array}\right), \quad M^{\top}=\left(\begin{array}{cc}
B^{\top} & A^{\top} \\
-I & 0 \\
I & 0
\end{array}\right), \quad M^{\top} y=\left(\begin{array}{c}
B^{\top} y_{0}+A^{\top} y_{b} \\
-y_{0} \\
y_{0}
\end{array}\right),
$$

this reads

$$
\min _{y_{0}, y_{b}}-\left\langle b, y_{b}\right\rangle \quad \text { s.t. } \quad B^{\top} y_{0}+A^{\top} y_{b} \leq 0, \quad-1 \leq y_{0} \leq 1 .
$$

Moreover, both primal and dual solutions $(\bar{x}, \bar{y})$ will satisfy the following optimality conditions

$$
\begin{gathered}
0 \leq c-M^{\top} y \perp x \geq 0, \\
M x=q .
\end{gathered}
$$

\subsection{Uniqueness of Primal LP}

A classical argument for replacing $\|\cdot\|_{0}$ by $\|\cdot\|_{1}$ and solving for (6) is uniqueness of the minimal $\ell_{1}$ (thus LP) solution. Let $\bar{x}=(\bar{u}, \bar{v})=\left(\bar{u}, \bar{v}^{1}, \bar{v}^{2}\right)$ be $\ell$-cosparse and solve (9). We assume throughout

$$
\bar{u}_{i}>0, i \in[n] .
$$

Based on $\bar{x}$, we define the corresponding support set

$$
J:=\left\{i \in[\operatorname{dim}(x)]: \bar{x}_{i} \neq 0\right\}=\operatorname{supp}(\bar{x}), \quad \bar{J}:=J^{c}=[\operatorname{dim}(x)] \backslash J .
$$


Denoting $k:=p-\ell$, the cardinality of the index sets $J$ and $\bar{J}$ is

$$
|\bar{J}|=2 \ell+k=p+\ell, \quad|J|=n+2 p-|\bar{J}|=n+k,
$$

compare [2, Lem. 5.2]. This shows that $x \in \mathbb{R}^{n+2 p}$ is a $(n+k)$-sparse vector.

Theorem 1 ([6, Thm. 2(iii)]). Let $\bar{x}$ be a solution of the linear program (9). The following statements are equivalent:

(i) $\bar{x}$ is unique.

(ii) There exists no $x$ satisfying

$$
M x=0, \quad x_{\bar{J}} \geq 0, \quad\langle c, x\rangle \leq 0, \quad x \neq 0 .
$$

Theorem (11) can be turned into a nullspace condition w.r.t. the sensor matrix $A$, for the unique solvability of problems (9) and (6).

Proposition 2 ([2, Cor. 5.3]). Let $\bar{x}=\left(\bar{u}, \bar{v}^{1}, \bar{v}^{2}\right)$ be a solution of the linear program (9) with component $\bar{u}$ that has cosupport $\Lambda$ with respect to $B$. Then $\bar{x}$, resp. $\bar{u}$, are unique if and only if

$$
\forall x=\left(\begin{array}{l}
u \\
v
\end{array}\right), v=\left(\begin{array}{l}
v^{1} \\
v^{2}
\end{array}\right) \quad \text { s.t. } \quad u \in \mathcal{N}(A) \backslash\{0\} \quad \text { and } \quad B u=v^{1}-v^{2}
$$

the condition

$$
\left\|(B u)_{\Lambda}\right\|_{1}>\left\langle(B u)_{\Lambda^{c}}, \operatorname{sign}(B \bar{u})_{\Lambda^{c}}\right\rangle
$$

holds. Furthermore, any unknown $\ell$-cosparse vector $u^{*}$, with $A u^{*}=b$, can be uniquely recovered as solution $\bar{u}=u^{*}$ to (6) if and only if, for all vectors $u$ conforming to (18), the condition

$$
\left\|(B u)_{\Lambda}\right\|_{1}>\sup _{\Lambda \subset[p]:|\Lambda|=\ell} \sup _{\bar{u} \in \mathcal{N}\left(B B_{\Lambda}\right)}\left\langle(B u)_{\Lambda^{c}}, \operatorname{sign}(B \bar{u})_{\Lambda^{c}}\right\rangle
$$

holds.

Remark 1. Conditions (19) and (20) clearly indicate the direct influence of cosparsity on the recovery performance: if $\ell=|\Lambda|$ increases, then these conditions will more likely hold. On the other hand, these results are mainly theoretical since numerically checking (20) is infeasible. However, we will assume that uniqueness of (6) is given, provided that the cosparsity $\ell$ of the unique solution $\bar{u}$ satisfies the conditions in (5a) and (5b). This assumption is motivated by the comprehensive experimental assessment of recovery properties reported in [2].

Remark 2. We note that, besides the condition for uniqueness from Thm. (1), a LP solution is unique if there is a unique feasible point. For high cosparsity levels $\ell$, this seems to be often the case.

Let $\bar{x}$ be a (possibly unique) primal solution of $(P)$ and $\bar{y}$ a dual solution. In view of (15) and (12) we have

$$
\left(c-M^{\top} \bar{y}\right)_{i}=0, \quad \forall i \in J .
$$

We note that non-degeneracy of the primal-dual pair $(\bar{x}, \bar{y})$ implies uniqueness of the dual variable $\bar{y}$. 


\section{Recovery by Perturbed Linear Programming}

Preliminaries: Fenchel Duality Scheme. We will use the following result.

Theorem 2 ([5]). Let $f: \mathbb{R}^{n} \rightarrow \mathbb{R} \cup\{+\infty\}, g: \mathbb{R}^{m} \rightarrow \mathbb{R} \cup\{+\infty\}$ and $A \in$ $\mathbb{R}^{m \times n}$. Consider the two problems

$$
\begin{array}{ll}
\inf _{x \in \mathbb{R}^{n}} \varphi(x), & \varphi(x)=\langle c, x\rangle+f(x)+g(b-A x), \\
\sup _{y \in \mathbb{R}^{m}} \psi(y), & \psi(y)=\langle b, y\rangle-g^{*}(y)-f^{*}\left(A^{\top} y-c\right) .
\end{array}
$$

where the functions $f$ and $g$ are proper, lower-semicontinuous (lsc) and convex. Suppose that

$$
\begin{aligned}
& b \in \operatorname{int}(A \operatorname{dom} f+\operatorname{dom} g), \\
& c \in \operatorname{int}\left(A^{\top} \operatorname{dom} g^{*}-\operatorname{dom} f^{*}\right) .
\end{aligned}
$$

Then the optimal solutions $\bar{x}, \bar{y}$ are determined by

$$
0 \in c+\partial f(\bar{x})-A^{\top} \partial g(b-A \bar{x}), \quad 0 \in b-\partial g^{*}(\bar{y})-A \partial f^{*}\left(A^{\top} \bar{y}-c\right)
$$

and connected through

$$
\begin{aligned}
\bar{y} & \in \partial g(b-A \bar{x}), & \bar{x} & \in \partial f^{*}\left(A^{\top} \bar{y}-c\right), \\
A^{\top} \bar{y}-c & \in \partial f(\bar{x}), & b-A \bar{x} & \in \partial g^{*}(\bar{y}) .
\end{aligned}
$$

Furthermore, the duality gap vanishes: $\varphi(\bar{x})=\psi(\bar{y})$.

Entropic Perturbation and Exponential Penalty. In various approaches to solving large-scale linear programs, one regularizes the problem by adding to the linear cost function a separable nonlinear function multiplied by a small positive parameter. Popular choices of this nonlinear function include the quadratic function, the logarithm function, and the $\langle x, \log (x)\rangle$-entropy function. Our main motivation in following this trend is that by adding a strictly convex and separable perturbation function, the dual problem will become unconstrained and differentiable. Consider

$$
\left(P_{\varepsilon}\right) \quad \min \langle c, x\rangle+\varepsilon\langle x, \log x-\mathbb{1}\rangle \quad \text { s.t. } \quad M x=q, x \geq 0 .
$$

The perturbation approach by the entropy function was studied by Fang et al. [4/7] and, from a dual exponential penalty view, by Cominetti et al. [8].

The Unconstrained Dual. We write $\left(P_{\varepsilon}\right)(26)$ in the form (22a)

$$
\min \varphi(x), \quad \varphi(x):=\langle c, x\rangle+\underbrace{\varepsilon\langle x, \log x-\mathbb{1}\rangle+\delta_{\mathbb{R}_{+}^{n}}(x)}_{:=f(x)}+\delta_{0}(q-M x) .
$$


With $g:=\delta_{0}$, we get $g^{*} \equiv 0$, since $\delta_{C}^{*} \equiv \sigma_{C}$ and thus

$$
g^{*}(y)=\delta_{0}^{*}(y)=\sigma_{0}(y)=\sup _{z=0}\langle y, z\rangle=0, \quad \forall y \in \mathbb{R}^{n}
$$

holds. On the other hand, we have $f^{*}(y)=\varepsilon\left\langle\mathbb{1}, e^{\frac{y}{\varepsilon}}\right\rangle$. Now (22b) gives immediately the dual problem

$$
\sup \psi(y), \quad \psi(y):=\langle q, y\rangle-\varepsilon\left\langle\mathbb{1}, e^{\frac{M^{\top} y-c}{\varepsilon}}\right\rangle .
$$

We note that $\psi$ is unconstrained and twice differentiable with

$$
\begin{aligned}
\nabla \psi(y) & =q-M e^{\frac{M^{\top} y-c}{\varepsilon}} \quad \text { and } \\
\nabla^{2} \psi(y) & =-\frac{1}{\varepsilon} M \operatorname{diag} e^{\frac{M^{\top} y-c}{\varepsilon}} M^{\top} .
\end{aligned}
$$

Moreover, $-\nabla^{2} \psi \succ 0$ for all $y$, with $e^{\frac{M^{\top} y-c}{\varepsilon}} \in \mathcal{R}(M)=\mathcal{N}(M)^{\perp}$, in view of (29b). Note that if $\psi$ has a solution, then it is unique and the strictly feasible set must be nonempty, see (29a), thus $\operatorname{rint} \mathcal{P}=\{x: M x=q, x>0\} \neq \emptyset \Leftrightarrow$ $q \in M\left(\mathbb{R}_{++}^{n}\right)$. Further, we can rewrite (28) in a more detailed form in view of (10)

$$
\left(D_{\varepsilon}\right) \quad \min _{y_{0}, y_{b}}-\left\langle b, y_{b}\right\rangle+\varepsilon\left\langle\mathbb{1}_{n}, e^{\frac{B^{\top} y_{0}+A^{\top} y_{b}}{\varepsilon}}\right\rangle+\varepsilon\left\langle\mathbb{1}_{p}, e^{\frac{-y_{0}-\mathbb{1}_{p}}{\varepsilon}}\right\rangle+\varepsilon\left\langle\mathbb{1}_{p}, e^{\frac{y_{0}-\mathbb{1}_{p}}{\varepsilon}}\right\rangle .
$$

Connecting Primal and Dual Variables. With $\operatorname{dom} g=0, \operatorname{dom} g^{*}=\mathbb{R}^{n}$, $\operatorname{dom} f^{*}=\mathbb{R}^{n}$ and $\operatorname{dom} f=\mathbb{R}_{+}^{n}$, the assumptions (23) become $q \in \operatorname{int} M\left(\mathbb{R}_{+}^{n}\right)=$ $M\left(\right.$ int $\left.\mathbb{R}_{+}^{n}\right)=M\left(\mathbb{R}_{++}^{n}\right)$, compare [5, Prop. 2.44], and $c \in$ int $\mathbb{R}^{n}=\mathbb{R}^{n}$. Thus, under the assumption of a strictly feasible set, we have no duality gap. Moreover both problems (27) and (28) have a solution.

Theorem 3. Denote by $x_{\varepsilon}$ and $y_{\varepsilon}$ a solution of $\left(P_{\varepsilon}\right)$ and $\left(D_{\varepsilon}\right)$ respectively. Then the following statements are equivalent:

(a) $q \in M\left(\mathbb{R}_{++}^{n}\right)$, thus the strictly feasible set is nonempty.

(b) The duality gap is zero $\psi\left(y_{\varepsilon}\right)=\varphi\left(x_{\varepsilon}\right)$.

(c) Solutions $x_{\varepsilon}$ and $y_{\varepsilon}$ of $\left(P_{\varepsilon}\right)$ and $\left(D_{\varepsilon}\right)$ exist and are connected through

$$
x_{\varepsilon}=e^{\frac{M^{\top} y_{\varepsilon}-c}{\varepsilon}} \text {. }
$$

Proof. (a) $\Rightarrow(\mathrm{b})$ : holds due to Thm. 2, On the other hand, (b) implies solvability of $\psi$ and thus (a), as noted after Eq. (29b). (a) $\Rightarrow(\mathrm{c})$ : The assumptions of Thm.2 hold. Now $\partial f^{*}(y)=\left\{\nabla f^{*}(y)\right\}=\left\{e^{\frac{y}{\varepsilon}}\right\}$ and the r.h.s. of (25a) gives (c). Now, (c) implies $M x_{\varepsilon}=q$ and thus (a).

The following result shows that for $\varepsilon \rightarrow 0$ and under the nonempty strictly feasible set assumption, $x_{\varepsilon}$ given by (31) approaches the least-entropy solution of $(P)$, if $y_{\varepsilon}$ is a solution of $\left(D_{\varepsilon}\right)$. The proof follows along the lines of [9, Prop. 1$]$. 
Theorem 4. Denote the solution set of (9) by $\mathcal{S}$. Assume $\mathcal{S} \neq \emptyset$ and $\operatorname{rint} \mathcal{P} \neq \emptyset$. Then, for any sequence of positive scalars $\left(\varepsilon_{k}\right)$ tending to zero and any sequence of vectors $\left(x_{\varepsilon_{k}}\right)$, converging to some $x^{*}$, we have $x^{*} \in \operatorname{argmin}_{x \in S}\langle x, \log x-\mathbb{1}\rangle$. If $\mathcal{S}$ is a singleton, denoted by $\bar{x}$, then $x_{\varepsilon_{k}} \rightarrow \bar{x}$.

Partial Perturbation. In the case of a unique and sparse feasible point $\bar{x}$ the assumption $q \in M\left(\mathbb{R}_{++}^{n}\right)$ does not hold. With $J=\operatorname{supp}(\bar{x})$ the primal reads

$$
\min \langle c, x\rangle+\varepsilon\left\langle x_{J}, \log x_{J}-\mathbb{1}_{J}\right\rangle \quad \text { s.t. } \quad M x=q, x_{J^{c}}=0, x \geq 0,
$$

and the dual becomes

$$
\max _{y}\langle q, y\rangle-\varepsilon\left\langle\mathbb{1}, e^{\frac{\left(M^{\top}\right)_{J} y-c_{J}}{\varepsilon}}\right\rangle .
$$

However, the solution support $J$ is unknown. Using (21), one can show that an approximative solution $y_{\varepsilon}$ of $\left(D_{\varepsilon}\right)$, i.e. $\left\|\nabla \psi\left(y_{\varepsilon}\right)\right\| \leq \tau_{\varepsilon}$, with $\tau_{\varepsilon}>0$ small, can be used to construct $x_{\varepsilon}$ according to (31), such that $x_{\varepsilon} \rightarrow \bar{x}$.

Exponential Penalty Method. We discussed above how problem $\left(P_{\varepsilon}\right)$ tends to $(P)$ as $\varepsilon \rightarrow 0$. Likewise, $\left(D_{\varepsilon}\right)$ tends to $(D)$. This was shown by Cominetti et al. [8, Prop. 3.1]. The authors noticed that the problem $\left(D_{\varepsilon}\right)$ is a exponential penalty formulation of $(D)$, compare (10) and (30).

They also investigated the asymptotic behavior of the trajectory $y_{\varepsilon}$ and its relation with the solution set of $(D)$. They proved the trajectory $y_{\varepsilon}$ is approximatively a straight line directed towards the center of the optimal face of $(D)$, namely $y_{\varepsilon}=y^{*}+\varepsilon d^{*}+\eta(\varepsilon)$, where $y^{*}$ is a particular solution of $(D)$. Moreover, the error $\eta(\varepsilon)$ goes to zero exponentially fast, i.e. at the speed of $e^{\frac{-\mu}{\varepsilon}}$ for some $\mu>0$. See the proof of [8, Prop. 3.2].

\section{$5 \quad$ Numerical Experiments}

In this section, we illustrate the performance of our perturbation approach compared to the LP solver MOSEK, in noisy and non-noisy environments, for $2 \mathrm{D}$ and $3 \mathrm{D}$ cases. Besides the proposed entropic approach, we implemented a quadratic perturbation approach for comparison purposes. We solved the perturbed dual formulations by a conventional unconstrained optimization approach, the Limited Memory BFGS algorithm [10, which scales to large problem sizes. In all experiments, the perturbation parameters were kept fixed to $\varepsilon=1 / 50$ and $\alpha=1$, see Fig. 3 for a justification. In the following $\frac{1}{2 \alpha}$ denotes the perturbation parameter of a quadratic term applied to (6). We allowed a maximum number of 1500 iterations and stopped when the norm of the gradient of the perturbed dual function satisfies $\left\|\nabla \psi\left(y^{k}\right)\right\| \leq 10^{-4}$.

The first performance test was done on $2 \mathrm{D} d \times d$ images of randomly located ellipsoids with random radii along the coordinate axes. See Fig. 1 (bottom row) 

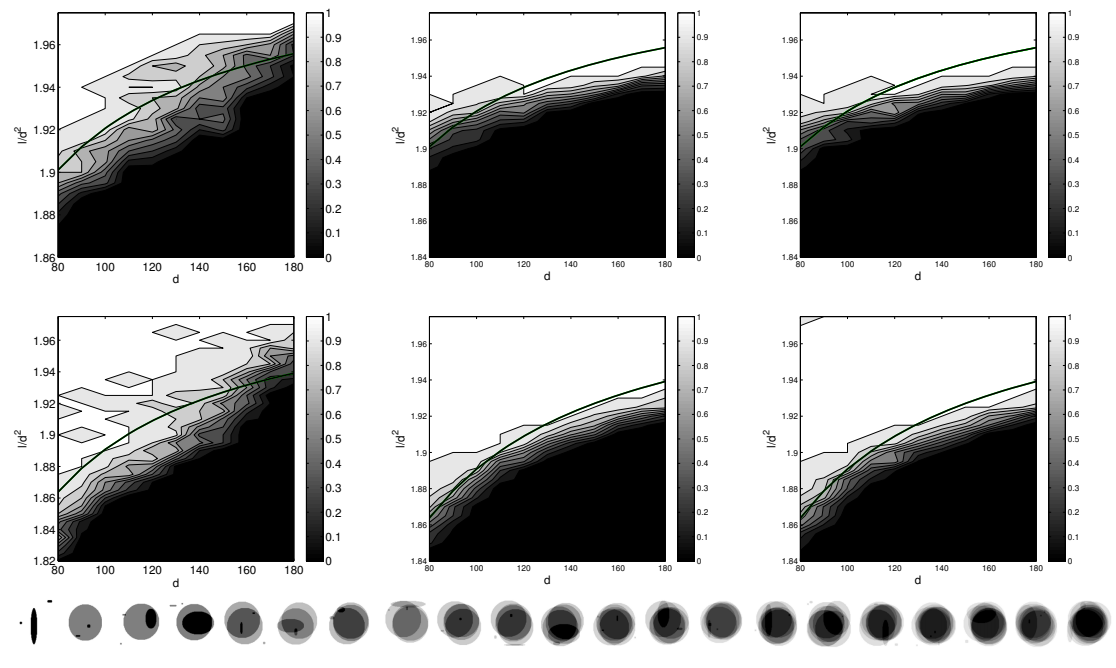

Fig. 1. Phase transitions for the 2D case, $d=80: 10: 180,4$ cameras (top plots) and 6 cameras (bottom plots), computed for the noiseless case with MOSEK (plots on left), our approach (plots in the middle) and our approach for the noisy case (plots on the right). The black solid line corresponds to the theoretical curve (5a). The last row displays some of the random $d \times d$ images used in the experiments in order of decreasing cosparsity $\ell$. MOSEK performs better on smaller images and worse on larger ones.
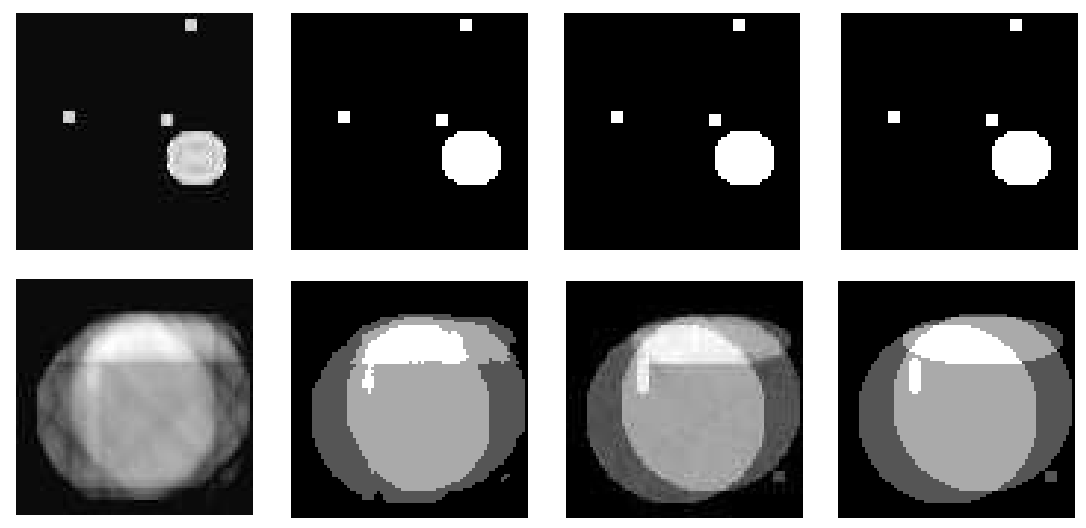

Fig. 2. Comparison between the quadratic perturbation approach (left two columns) and entropic perturbation approach (right two columns) for two relative cosparsity levels. Two $80 \times 80$ images, are projected along 6 directions. For both $\rho=\ell / d^{2}=1.7786$ (top row) and $\rho=\ell / d^{2}=1.8586$ (bottom row) reconstruction should in theory be exact. Result (left column) and rounded result (second left column) of the quadratic perturbation approach for $\alpha=1$. Results for the entropic perturbation approach (right two columns) with $\varepsilon=1 / 50$. Here the rounded result exactly equals the original image (right column). Hence, the approximate solution by the entropic approach is closer to the original solution. 

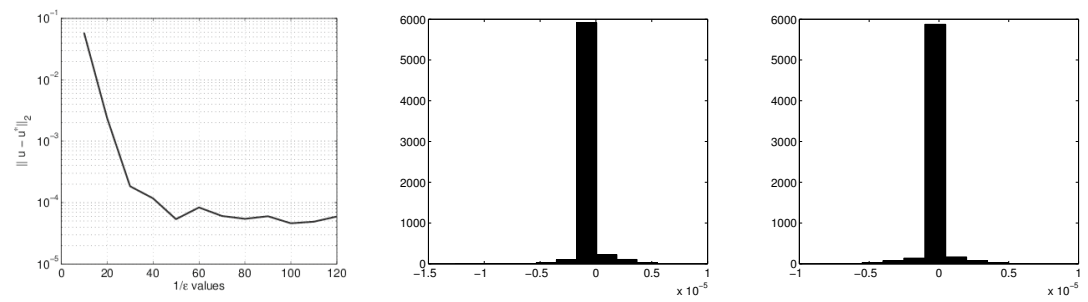

Fig. 3. Experimental finite perturbation property of the entropic approach. Here $\varepsilon=$ $1 / 50$ is a reasonable value in $2 \mathrm{D}$ since the reconstruction error varies insignificantly (left). The histograms of $\left(u-u^{*}\right)$ for $\varepsilon=1 / 50$ (middle) and $\varepsilon=1 / 120$ (right) are highly similar.
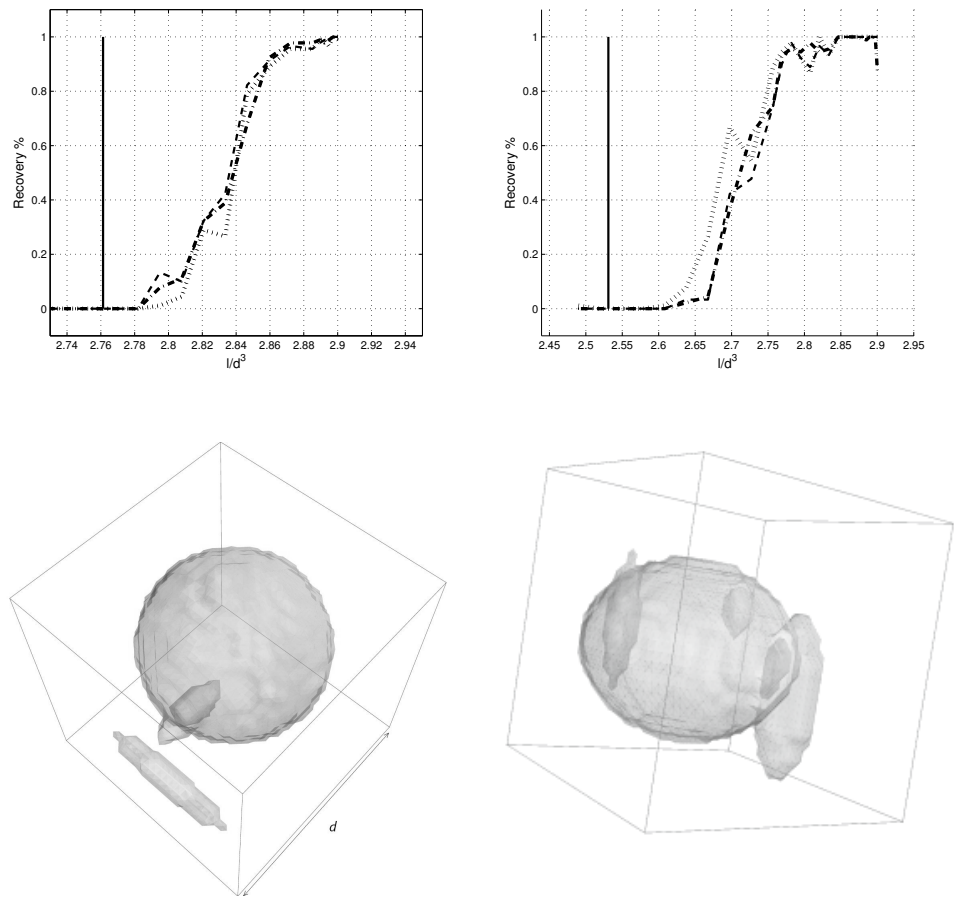

Fig. 4. Phase transitions for the 3D case, 3 cameras (top left) and 4 cameras (top right) and random example of perfectly reconstructed images $d=31$ (bottom). The average performance of MOSEK (dotted line) for the noiseless case, and the entropic approach in the noiseless (dashed line) and noisy (dash-dot line) case for $\varepsilon=1 / 50$ as a variation of relative cosparsity. The black solid line corresponds to the theoretical curve (5b). Measurements were corrupted by Poisson noise of SNR $=50 \mathrm{db}$. 

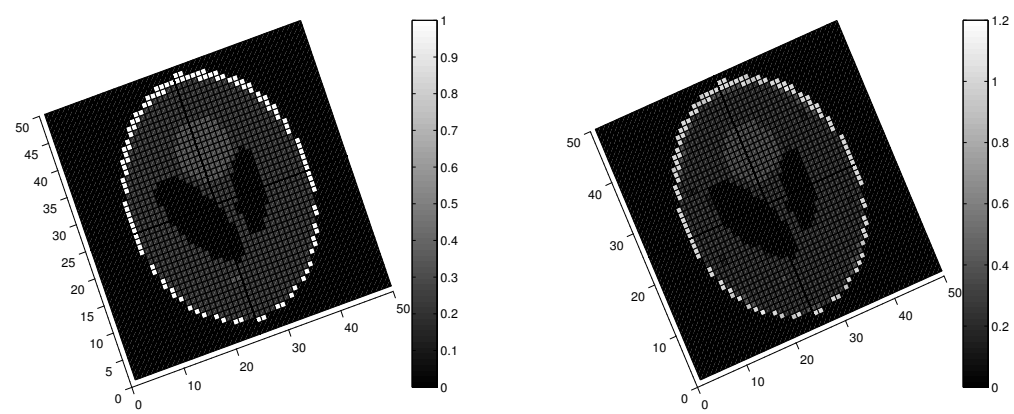

Fig. 5. Slices through the 3D volume $(d=51)$ of an original Shepp-Logan image (left) and the reconstructed image from 7 noisy projecting directions via the entropic perturbation approach, satisfying $\left\|u-u^{*}\right\|_{\infty}<0.5$ (right). This shows that the approach is also stable for low noise levels as opposed to MOSEK. Measurements were corrupted by Poisson noise of $\mathrm{SNR}=50 \mathrm{db}$.

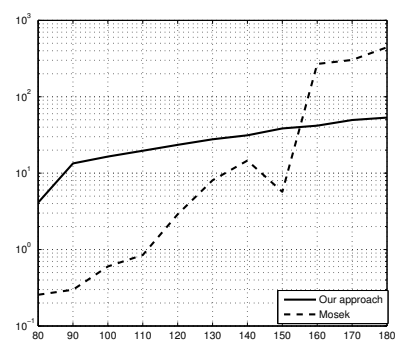

Fig. 6. Comparison between computation times of the proposed approach and MOSEK. We average results for $902 \mathrm{D}$ test images and vary $d=80: 10: 180$.

for two sample images. The relative cosparsity is denoted by $\rho:=\frac{\ell}{n}$. Parameters $p$ and $n$ vary for two- and three-dimensional images as

$$
n=\left\{\begin{array}{ll}
d^{2} & \text { in } 2 \mathrm{D} \\
d^{3} & \text { in } 3 \mathrm{D}
\end{array} \quad, \quad p=\left\{\begin{array}{ll}
2 d(d-1) & \text { in } 2 \mathrm{D} \\
3 d^{2}(d-1) & \text { in } 3 \mathrm{D}
\end{array} .\right.\right.
$$

Our parametrization relates to the design of the projection matrices $A \in \mathbb{R}^{m \times n}$, see 2 for details.

The phase transitions in Fig. 1 display the empirical probability of exact recovery over the space of parameters that characterize the problem. Here we performed 90 tests for each $(\rho, d)$ parameter combination.

We analyzed the influence of the image cosparsity, also for 3D images, see Fig. 4. In 3D, for each problem instance defined by a $(\rho, d)$-point, we generated 60 random images. In both $2 \mathrm{D}$ and $3 \mathrm{D}$, we declared a random test as successful 
if $\left\|u-u^{*}\right\|_{\infty}<0.5$, which leads to perfect reconstruction after rounding. Both Fig. 1 and Fig. 4 display a phase transition and exhibit regions where exact image reconstruction has probability equal or close to one and closely match the solid green line in the plots, which stands for the theoretical curve (5a). In the noisy case, projection data was corrupted by Poisson noise of $S N R=50 \mathrm{db}$. The perturbation parameter has been set as in the noiseless case, i.e. $\varepsilon=1 / 50$ and $\alpha=1$. MOSEK however was unable to solve the given problem, stating that either the primal or the dual might be infeasible. Thus our perturbation approach is also stable to low noise levels as opposed to MOSEK. Moreover the proposed algorithm scales much better with the problem size and is significantly more efficient for large problem sizes that are relevant to applications. In particular, problem sizes can be handled where MOSEK stalls, see Fig. 6. Finally, we underline that the entropic perturbation approach performs significantly better than quadratic perturbation as shown in Fig. 2.

\section{Conclusion}

We presented a mathematical programming approach based on perturbation that copes with large tomographic reconstruction problems of the form (11). While the perturbation enables to apply efficient sparse numerics, it does not compromise reconstruction accuracy. This is a significant step in view of the big data volumes of industrial scenarios.

Our further work will examine the relation between the geometry induced by perturbations on the $u$-space and the geometry of Newton-like minimizing paths, and the potential for parallel implementations.

Acknowledgement. SP gratefully acknowledges financial support from the Ministry of Science, Research and Arts, Baden-Württemberg, within the Margarete von Wrangell postdoctoral lecture qualification program. AD and the remaining authors appreciate financial support of this project by the Bavarian State Ministry of Education, Science and Arts.

\section{References}

1. Nam, S., Davies, M., Elad, M., Gribonval, R.: The Cosparse Analysis Model and Algorithms. Applied and Computational Harmonic Analysis 34(1), 30-56 (2013)

2. Deniţiu, A., Petra, S., Schnörr, C., Schnörr, C.: Phase Transitions and Cosparse Tomographic Recovery of Compound Solid Bodies from Few Projections. ArXiv e-prints (November 2013)

3. Ferris, M.C., Mangasarian, O.L.: Finite perturbation of convex programs. Appl. Math. Optim. 23, 263-273 (1991)

4. Fang, S.C., Tsao, H.S.J.: Linear programming with entropic perturbation. Math. Meth. of OR 37(2), 171-186 (1993)

5. Rockafellar, R., Wets, R.J.B.: Variational Analysis, 2nd edn. Springer (2009)

6. Mangasarian, O.L.: Uniqueness of Solution in Linear Programming. Linear Algebra and its Applications 25, 151-162 (1979) 
7. Fang, S.C., Tsao, H.S.J.: On the entropic perturbation and exponential penalty methods for linear programming. J. Optim. Theory Appl. 89, 461-466 (1996)

8. Cominetti, R., San Martin, J.: Asymptotic analysis of the exponential penalty trajectory in linear programming. Math. Progr. 67, 169-187 (1994)

9. Tseng, P.: Convergence and Error Bound for Perturbation of Linear Programs. Computational Optimization and Applications 13(1-3), 221-230 (1999)

10. Bonnans, J.F., Gilbert, Lemaréchal, C., Sagastizábal, C.: Numerical Optimization - Theoretical and Practical Aspects. Springer, Berlin (2006) 